\title{
Analysis of Human Resource Allocation Model for Tourism Industry Based on Improved BP Neural Network
}

\author{
Shuo Zhu (iD ${ }^{1}$ and Yan Liu ${ }^{2}$ \\ ${ }^{1}$ Tourism College, Hebei University of Economics and Business, Shijiazhuang, Hebei 050061, China \\ ${ }^{2}$ Huihua College, Hebei Normal University, Shijiazhuang, Hebei 050000, China \\ Correspondence should be addressed to Shuo Zhu; zshbjmmice@heuet.edu.cn
}

Received 11 December 2021; Revised 25 December 2021; Accepted 27 December 2021; Published 12 January 2022

Academic Editor: Miaochao Chen

Copyright (c) 2022 Shuo Zhu and Yan Liu. This is an open access article distributed under the Creative Commons Attribution License, which permits unrestricted use, distribution, and reproduction in any medium, provided the original work is properly cited.

\begin{abstract}
This paper analyzes the deficiencies of human resource allocation in the tourism industry by investigating the human resource allocation in the tourism industry, puts forward corresponding improvement measures and suggestions, and strives to provide certain guidance and helpful effects for the construction of tourism resource informatization. In this paper, a modified BP neural network model is proposed by introducing random perturbation terms on the hidden layer in the BP neural network algorithm, and the weight matrix connecting the input values is added with the random perturbation matrix to obtain a new weight matrix so that the convergence effect of the improved BP neural network algorithm is improved. Then, to address the problem that the initial weights of the long and short-term memory neural network and gated BP unit neural network have a large impact on the convergence speed and prediction accuracy of the algorithm after the initial weight selection is determined, this paper introduces the random perturbation term into the gate structure of the long and short-term memory neural network and gated BP unit neural network and proposes and connects an improved long and short-term memory neural network and gated BP unit neural network. The weight matrix of the input values is added with the random perturbation matrix to obtain the new weight matrix so that the convergence effect of the improved long and short-term memory neural network algorithm and the gated BP unit neural network algorithm is improved. Constructing the human resource allocation model of the tourism industry and proposing coping strategies and countermeasures and taking the human resource allocation system of the tourism industry as the core, the human resource allocation model of the tourism industry is established by combining the network image crisis life cycle system of tourism scenic spots and the network public opinion dissemination model. From the perspective of managers, the human resource allocation management policy and management procedures of the tourism industry are proposed. Using the quantifiable and disenable characteristics of online text information, the response strategy of online monitoring and propaganda and offline management and enhancement is proposed, and innovative countermeasures to the human resource allocation of the tourism industry are proposed in three categories: network originated, reality coexisting, and reality originated. Through this paper, we propose a new approach to human resource allocation management and development in the tourism industry and improve the efficiency of human resource allocation in the tourism industry.
\end{abstract}

\section{Introduction}

With the acceleration of the global economic integration process, human resources have gradually become core resources. After defining human resources as a strategy for a strong nation, it has been positioned as a basic national strategy, which shows the deepening importance attached to human resources. With the deepening of reform and opening up and the development of economic construction, it has become urgent to strengthen human resources development, human resources' capacity building, and human resources' policy research [1]. Tourism human resources determine the development of the tourism industry, so cultivating the raw power of the tourism industry and attaching great importance to tourism human resources will be an important and arduous task for the development of China's tourism industry. Tourism human resources are complex social system engineering, which include both the 
tourism human resources that are often in direct contact with tourists and the tourism human resources that are indirectly in contact with tourists. Tourism resources are extremely rich and unique; tourism, as its support industry, has become an important way and fundamental guarantee for the rapid development of the regional economy and the full implementation of well-off [2]. Tourist attractions are the basic building blocks of tourist destinations, and the quality of their network image directly affects potential tourists' perception of the destination's image, which in turn affects their behavioral decisions and jeopardizes the business performance of tourist attractions as well as the strategic goal of sustainable development. However, there is a lack of attention to this aspect in tourist attractions at present. Tourist attractions mostly focus on daily management and simple advertising, lacking an overall awareness of human resources allocation in tourism, control of the inner mechanism of the network image of tourist attractions, and marketing strategies and lacking a sense of worry about human resources allocation in tourism [3].

Human resources, as the carrier of knowledge, have become the target of enterprises. Under the influence of the people-oriented concept, the role of human resources' management in tourism enterprises has become increasingly prominent. Human resources' development and management is the use of modern scientific methods, with a certain combination of human resources for training, human and material resources' organization, and deployment to maintain the best ratio, while the human mind psychological behavior induction also gives full play to the initiative and appropriate personnel, control, and cooperation with the appropriate to achieve the goals of the organization [4]. This study intends to study the development and management of human resources in the tourism industry, through comparative analysis, to clarify some experiences and recommendations for better development and management of human resources in the tourism industry in the future and to provide guidance and reference for human resources in the tourism industry. In the web text data collection, the web crawler software is used to capture the text information, and NLPIR-ICTCLAS is used to carry out the subword statistics, which enriches the collection and measurement tools and research tools for the image of tourism scenic spots, increases the data transformation methods in this research field, realizes the combination of qualitative research and quantitative research, and lays the foundation for other quantitative research on human resource allocation in the tourism industry. In addition, the application of the BP neural network model provides a new paradigm for the design of human resource allocation models in tourism, broadens the research field of human resource allocation in tourism, and extends the application of MATLAB mathematical software in the field of tourism crisis management $[5,6]$.

The purpose of this paper is to put forward a more perfect human resource allocation system and management mode from the perspective of human resource allocation, to improve the awareness of the management of tourist attractions and strengthen the cognition of the network image of tourist attractions and to effectively provide a realistic management problem for the expansion of tourist attractions in the context of the changing times of the network era [7]. To improve the awareness of tourism scenic spot management, strengthen the awareness of tourism scenic spot network image; to effectively provide a point of force to expand the real operation problems of tourism scenic spot, realize the sustainable development of tourism scenic spot and, at the same time, provide an important role in promoting the transformation and upgrading of the modern tourism industry. And the improved BP neural network algorithm is applied to the human resource allocation problem in the tourism industry. Compared with the version before the improvement, the improved BP neural network algorithm converges faster and is less likely to converge to the local optimal solution. Section 1 briefly describes the importance of studying the improved BP neural network for human resource allocation model analysis in the tourism industry, which has strong economic significance and practical value and finally introduces the main contents of this paper. Section 2 introduces the current situation of human resource allocation research in the tourism industry at home and abroad and the research directions around the BP neural network and its improvement. Section 3 studies the human resource allocation model of the tourism industry based on the improved BP neural network and gives the theoretical derivation. For the problem of BP neural network due to the large randomness of initial weight selection, the procedure of BP neural network algorithm based on random perturbation is designed by adding a random perturbation term to the hidden layer of BP neural network and applied to the human resource allocation problem of the tourism industry. Section 4 conducts the result analysis, and the experimental results show that the improved BP neural network can make the influence of initial weights on the convergence speed of the algorithm reduced and prevent the occurrence of the overfitting phenomenon. Section 5 first summarizes the work done and the proposed methods in this paper, then analyzes the methods and experiments proposed in the paper for improvement, and finally provides an outlook for future research work.

\section{Related Work}

Tourism human resources are an important guarantee to enhance the competitiveness and sustainable development of tourism and determine the development of tourism. With the further development of China's tourism industry, tourism human resources' development and management have become an important issue in the overall development of the tourism industry. Most of the research on the development and management of the tourism industry starts from the perspective of human resources of tourism enterprises, and less considers the mobility and turnover rate of tourism practitioners [8]. Shetaban et al. established a model based on a BP neural network-based analysis against the main components, as a way to achieve the rating of the degree of managerial defense of managers [9]. Ronoud and Asadi studied the economic evaluation of power transmission 
projects based on a fruit fly optimized BP neural network model, followed by the application of socio-economic development evaluation and assisted decision-making, and the applied research in this field more fully reflects the powerful performance of BP neural networks in terms of no linearization and self-learning habits to make scientific measurements of sustainable development and planning problems [10]. By using the impact degree model and sensitivity model of the BP neural network, Aakash et al. analyzed the relevant index data to derive the regional development constraints. From the perspective of tourism crisis early warning, constructing the network image impact index system of tourism scenic spots, extracting data samples by using methods such as network text content analysis, constructing BP neural network model, and then making analysis of the early warning system, there is still a gap in the research in this area [11]. Therefore, based on the relevant literature research and theoretical analysis, this paper innovatively proposes the human resource allocation system of the tourism industry, and based on the relevant literature and theoretical analysis, this paper proposes an innovative human resource allocation system for the tourism industry, analyzes the relevant data, and establishes a BP neural network model under the condition that the indicators are tested and corrected [12].

BP neural networks have made progress in areas such as human resources in the tourism industry because of their ability to predict time-series data. Since the emergence of BP neural networks, many scholars at home and abroad have studied and applied BP neural networks and their improvements [13]. Cheng and Zhao improved the activation function of RNN, which effectively accelerated the convergence speed of training [14]. Kang et al. simplified the highway BP neural network based on which the relevant parameters were significantly reduced and the computational complexity was reduced [15]. Dai and $\mathrm{Hu}$ proposed a quantum weighting method for BP neural networks with better computational efficiency. Theoretical research in tourism is not compatible with the development of the tourism industry, and the level of academic research and theoretical development is lower than that of the tourism industry [16]. Convolutional neural networks have gradually become a research hotspot in many fields in recent years. To achieve a better role in these fields and provide more convenience for people's daily life, it is necessary to get better training results [17]. This paper focuses on evaluating the classification of convolutional neural networks from a new perspective and improving the training method of convolutional neural networks after making relevant evaluations to make full use of the training data and obtain better classification results. Using knowledge consistency as a tool for diagnosing neural network representations and providing new insights to explain the success of existing deep learning techniques, knowledge consistency can also be used to improve pretrained networks and enhance performance [18]. The work in this paper uses the interclass distance of feature maps to understand the discriminatory ability of the trained model between different classes and improves the training process through analysis, providing a new perspective to improve the classification effectiveness of CNNs [19].

The scope of research on tourism-related theories is relatively small, and the main findings of this paper describe the methodology of the main study of numerical statistics is small. Most of them are from the research universities and other universities; governments and enterprises have given little support. The scope of its research is relatively narrow, mostly for the study of traditional industries, tourism products, human resources, tourism markets, and tourism planning, and other aspects still need to carry out the urgent need for systematic research work [20]. Due to the slow development of tourism theory research, many issues in the process of tourism development cannot be used as a basis and guidance. Therefore, the government should strengthen the macromanagement of tourism research, the development of the tourism industry, and encourage enterprises to actively participate in the system of combining academic research and tourism industry, the development of tourism industry, and tourism theory and improving the training of convolutional neural networks using validation set and distance metric. By combining the tourism scenic network image crisis warning index and the research problem, a three-layer BP neural network model is designed, which is commonly referred to as a classical BP neural network model including three layers: input layer, hidden layer, and output layer. After normalizing the sample data, the number of nodes in each layer is determined, and the model of the human resource allocation system for the tourism industry is simulated and experimented with using MATLAB software. Introducing BP neural network information technology into the human resource allocation of tourism industry, with the help of the real-time and high efficiency of the Internet, the human resource allocation of tourism industry is transformed from traditional manual management to intelligent platform management, and the management of human resource allocation of tourism industry is improved.

\section{Research on the Human Resource Allocation Model of Tourism Industry Based on Improved BP Neural Network}

3.1. Construction of Human Resource Allocation Parameters for the Tourism Industry. The entropy weight method can be used to scientifically and effectively calculate the weight coefficients between different indicators of tourism human resource allocation application and tourism development, and the results are more reliable. The shortcomings of the entropy weight method have less influence on the comprehensive level measurement of this paper and can scientifically assign weights to the results, which is a more objective evaluation method in general. With $m$ indicators and $n$ objects, the original data are arranged in rows to form a matrix (Equation (1)), and because the outline quantity of each evaluation indicator is different, the polar 
transformation method is used to standardize the original indicator data. Whenever an additional data point is obtained, the new point is added to the sample, and the oldest point in time is deleted, and then, the range related to this point is calculated. Therefore, the calculation of each range is at least. The calculation of the previous range shares the value of one point. The moving range is used for single value control charts, and usually, two points are used to calculate the moving range:

$$
H=\left(\begin{array}{cccc}
h_{11} & h_{12} & \cdots & h_{1 m} \\
h_{21} & h_{22} & \cdots & h_{2 m} \\
& \cdots & \ddots & \\
h_{n 1} & h_{n 2} & \cdots & h_{m n}
\end{array}\right) .
$$

Tourism demand level indicators are divided into 3 secondary indicators: total tourism revenue, total tourism receipts, and the proportion of total tourism revenue to the tertiary industry, total tourism revenue, and total tourism receipts; measure the scale and consumption capacity of the tourism market in general, and the proportion of total tourism revenue to the tertiary industry reflects the pulling effect of tourism demand on local economic and industrial development; the greater the pulling effect, the more it can improve local development of tourism. The greater the pull effect is, the more it can improve the local development of tourism enthusiasm, promote the transformation and upgrading of tourism, and promote tourism human resources allocation to better serve the development of tourism and to further better meet the demand for tourism, forming a virtuous development cycle.

The index system is developed from two levels of tourism human resources' allocation support and integration degree, and the evaluation of tourism human resources' allocation application development level in tourism industry is divided into two level indicators of tourism human resources' allocation support and integration degree, and the evaluation index system shown in Table 1 is constructed to measure the development level of tourism human resources' allocation application.

The first level of integration is divided into 2 secondary indicators, namely, Wi-Fi coverage of attractions and online tourism market transaction scale. The Wi-Fi coverage rate of attractions reflects the database of the tourism industry in the process of applying tourism human resource allocation to achieve its development and also reflects the convenience of using tourism human resource allocation application in the process of tourism, and the online tourism market transaction scale reflects the importance of tourism-related enterprises to tourism human resource allocation application, and a certain extent grasps the degree of integration and development of tourism and tourism human resource allocation application. The degree of integration of tourism and tourism HR application: Since the two types of indicators selected are of different outline quantities and are not uniform, the initial value transformation method is first used for normalization to form the data directly used for the calculation of gray correlation coefficients:

$$
\left\{\begin{array}{l}
A(x)=\lim _{N \rightarrow \infty} \sum_{i=1}^{N} \frac{A(x)}{A(i)} \\
x \subseteq[1, L] . \\
B(x)=\lim _{N \rightarrow \infty} \sum_{i=1}^{N} \frac{B(x)}{B(i)}
\end{array}\right.
$$

The gray correlation between each indicator of tourism human resource allocation application 5 in the tourism industry and the three primary indicators of tourism development is calculated:

$$
F(x)=\frac{\lim _{M, N \longrightarrow \infty} \sum_{i=1}^{M} \sum_{j=1}^{N} f(x)_{i j}}{m} .
$$

Modern tourism human resources need management, humanistic, and scientific talents. According to the survey, in tourism enterprises, there is a large shortage of management-level personnel in the economic, tourism, and foreign language fields, and the targeted training services and academic education provided by universities and colleges as well as a society cannot meet the development needs of many tourism industries. In the general workforce, only about $25 \%$ of the employees have a college degree or higher, and the proportion of tourism and related industries is even smaller, with a relatively low level of education, so it is necessary to increase the construction of professional personnel. From the government level, there is lack of tourism human resources overall scientific planning. Now, since the overall planning of tourism human resources is only lightly in the tourism planning, no specific talk should be on how to develop tourism human resources. This can almost be said to be in a state of coping with the lack of initiative and foresight in human resources planning work, which will certainly restrict the development of tourism human resources.

3.2. Improved BP Neural Network Human Resource Allocation Model for the Tourism Industry. The research in this paper focuses on the monitoring of the factors influencing the image of tourist attractions in the online environment for tourism human resource allocation. Therefore, the research object mainly involves the analysis and extraction of the content of information about the web text of the tourism scenic area's online image, which is used to form a factor model of tourism human resource allocation. Based on this, the factor model is based on the premise of distinguishing the sources of influence factors, the nature of influence factors, and the key of analyzing the specific influence contents as a whole, and the influence contents are specifically categorized into three major categories, namely, tourism resources, management level, and external environment, finally constructing the influence factor model of tourism human resource allocation, as shown in Figure 1. 
TABLE 1: The comprehensive evaluation system of tourism human resource allocation application development in the tourism industry.

\begin{tabular}{lcc}
\hline First-level indicator & Second-level indicator & Unit \\
\hline \multirow{2}{*}{ Human resources support A1 } & Software and information technology business revenue B1 & 1000 million yuan \\
& Internet penetration rate B2 & $\%$ \\
& Number of employees in electronic information industry B3 & Thousand people \\
\hline Integration degree A2 & Attraction WiFi coverage rate B4 & $\%$ \\
\end{tabular}

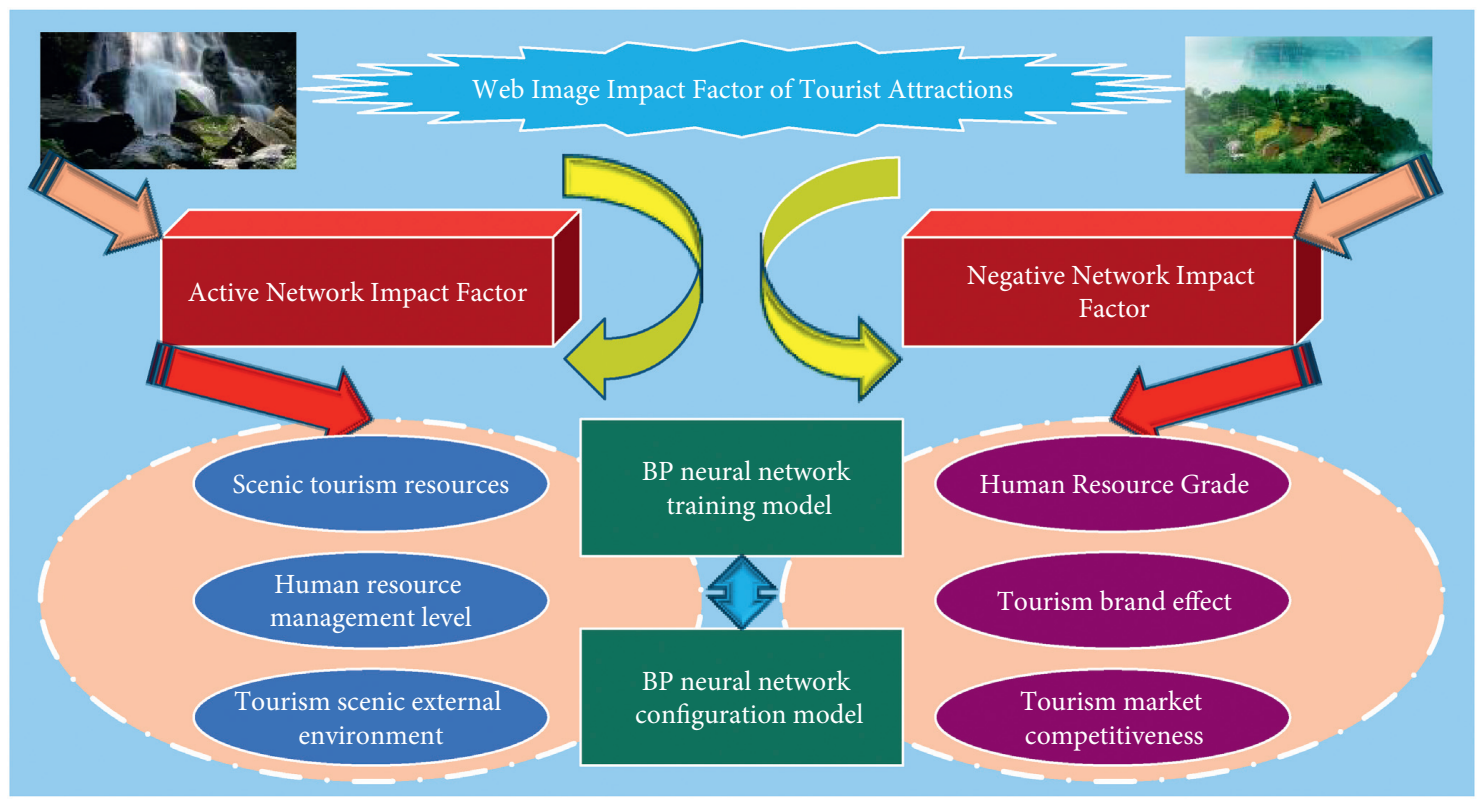

FIGURE 1: BP neural network model of the impact factor.

Based on the variability of the attributes of the indicators studied in this paper, all data involved in the reference equation (4) were normalized to lie within the interval $[-1,1]$ :

$$
A(x)=\frac{\left(A_{i}-\min \left(A_{i}\right)\right)\left(A_{i}+\max \left(A_{i}\right)\right)}{\max \left(A_{i}\right)-\min \left(A_{i}\right)}-1, i \subseteq[1, N] \text {. }
$$

In this paper, the input quantity, hidden layer nodes, and output layer are defined as the configuration indicators by combining the concepts related to human resource allocation in the tourism industry. Combined with the research data samples in this paper, the matrix is designed as

$$
M=\left(\begin{array}{cccc}
M_{11} & M_{12} & \cdots & M_{1 n} \\
M_{21} & M_{22} & \cdots & M_{2 n} \\
& \cdots & \ddots & \\
M_{n 1} & M_{n 2} & \cdots & M_{m n}
\end{array}\right), H=\left[h_{1}, h_{2}, \ldots \ldots h_{n}\right]^{T}
$$

Based on the correlation between the number of nodes in the hidden layer and the number of nodes in the input and output layers, the optimal number of nodes in the hidden layer is found according to the empirical formula (6). In equation (6), $i$ represents the number of samples, $M$ represents the number of nodes in the output layer, $m$ represents the number of nodes in the hidden layer, and $N$ represents the number of nodes in the input layer, combined with the "pooling method" to determine the final optimal number of nodes in the hidden layer:

$$
\left\{\begin{array}{l}
G=\lim _{N \longrightarrow \infty} \sum_{i=0}^{N} C_{i} \\
m=\ln N+\sqrt{N^{2}+M^{2}}+\beta
\end{array} .\right.
$$

At the same time, the improved BP neural network converges to a better local optimal solution than the traditional BP. It is known that the training set input data and training set output data are $M$ and $H$. A column of timeseries data $m_{1}, m_{2}, \ldots, m_{T}$ is represented as the value of $T$ time steps, where

$$
M(t)=\left[m_{1 t}, m_{2 t}, \ldots \ldots, m_{n t}\right]^{T} .
$$

The small-batch stochastic gradient descent algorithm is selected for weight update, i.e., the training set data $X$ and $Y$ are randomly disrupted into $S$ groups; each group consists of $J$ columns of time-series data. The input data and output data of the training set at time $t$ of the $j$ th column of the $S$ group can be expressed as

$$
\left\{\begin{array}{l}
S(x, t)=\left[m_{1 t}(x), m_{2 t}(x), \ldots \ldots, m_{n t}(x)\right]^{T} \\
S(y, t)=\left[n_{1 t}(y), n_{2 t}(y), \ldots \ldots, n_{n t}(y)\right]^{T}
\end{array} .\right.
$$

To make the improved BP converge faster, the parameter random perturbation term $\mu$ is introduced into the $\mathrm{BP}$ algorithm in this section, and the new weights are obtained by 
adding them with the input layer weights. The forward propagation process of the improved BP network is as

$$
S(t)=\beta\left(W(s) *\left(m_{t}(s), n_{t}(s)+a b\right) .\right.
$$

The activation function $\beta$ is the sigmoid function:

$$
\beta(x)=\frac{1}{1+\sum_{i=1}^{N} e^{-i}} .
$$

To ensure the randomness of the random perturbation parameters for each weight update, the backpropagation process only biases the original input layer weights. The improved BP network backpropagation process is shown as

$$
h(s, t)^{T}=\left(y(t)-\xi(t)_{M}^{N}\right) .
$$

The updated weights are calculated as

$$
\left\{\begin{array}{l}
w(h)=w(h)+\forall w(h) \\
b * w(s)=b * w(s)+\forall b * w(s)
\end{array} .\right.
$$

The objective function is found for the output value and the test set output data, as in equation (13), and the prediction error of the BP neural network is after the expectation of the objective function:

$$
E(x)=\sum_{i=1}^{M} \sum_{j=1}^{N}\left(x_{i j}-\phi_{i j}\right) .
$$

Using the idea of robust optimization methods, this paper comes to consider the worst case in all contexts, i.e., for the uncertainty set so that the model achieves the maximum of all minima, and because the uncertainty of customer arrival directly affects the penalty cost, the model becomes the following form:

$$
f(a, b, c)=\min \sum_{i=1}^{N}[w(a)+w(b)+w(c)] * l(t) .
$$

Equation (15) is the tourism human resource allocation model, which is a linear robust optimization model:

$$
\begin{aligned}
\min (F(a, b, c))= & \sum_{i=1}^{N} \alpha_{i} * w(a) \\
& +\sum_{i=1}^{N} \beta_{i} * w(b)+\sum_{i=1}^{N} \mu_{i} * w(c) .
\end{aligned}
$$

\subsection{Tourism Human Resource Allocation System Design.}

The tourism human resource allocation system is a complex large-scale WEB application system, and this system is developed using the multilayer framework structure of the $\mathrm{B} / \mathrm{S}$ model. The MVC model mainly corresponds to the part of the application server, the front-end corresponding to the user's request, the back-end responsible for the data transformation with the database server, and the middle is the application logic layer, responsible for the processing of specific business. In addition to the basic functions of the previous traditional tourism human resource allocation system, this paper further divides tourism resources into four categories of resources, which are related to the clothing, food, housing, and transportation of tourists. The personalized recommendation function of these four types of resources to users is realized based on adopting a unified data platform. The system architecture design diagram is shown in Figure 2. According to the connotation and characteristics of the neural network model of tourism resource informatization, follow the principles of scientificity, representativeness, independence, operability, purpose, completeness, and combination of qualitative and quantitative index selection.

Database design is based on E-R diagram and database model, detailed design of database fields and other attributes, detailed design of data tables, and data field information in the scenic management system, while ensuring the PostgreSQL database can store the type of data.

\section{Analysis of Results}

4.1. Human Resource Allocation Model Analysis. The interclass distance matrix and confusion matrix calculated on the test set using the traditional and improved models are shown in Figure 3. It can be seen that, with the same data, the number of correct classifications in the confusion matrix in Figure 3 is greatly increased and the interclass distances are larger, indicating that the classification ability of the model is effectively improved. This improved training method was validated on the EMNIST dataset using the improved BP neural network, and it was found that the improved method in this paper was effective, and it was also found that the interclass distances calculated on the learned feature maps could be used to improve the network training. The maximum value of the traditional model is slightly higher than the minimum value of $92.71 \%$ of the improved model, which proves that the accuracy of the model studied in this paper is higher.

After the training, the trained neural network model is used to input the values of each index of individual test samples into the model to obtain the output results shown in Figure 4. In neural network model research, the constructed model is usually used to test the test samples and calculate the relative error value between the actual evaluation results and the test results; if the relative error between the two is within the acceptable range, we consider that this indicates that the evaluation with the neural network model is effective and meets the requirements and can be used to evaluate the level of tourism resource informatization development.

4.2. Performance Analysis of the Improved Algorithm. The human resource data of the tourism industry was selected, and the BP algorithm was constructed using the Numpy library in Python software, and the BP neural network algorithm based on parameter random perturbation was constructed by adding a random perturbation term in the forward propagation process. The evaluation criterion of the experiment is the loss function of the test set, and the 


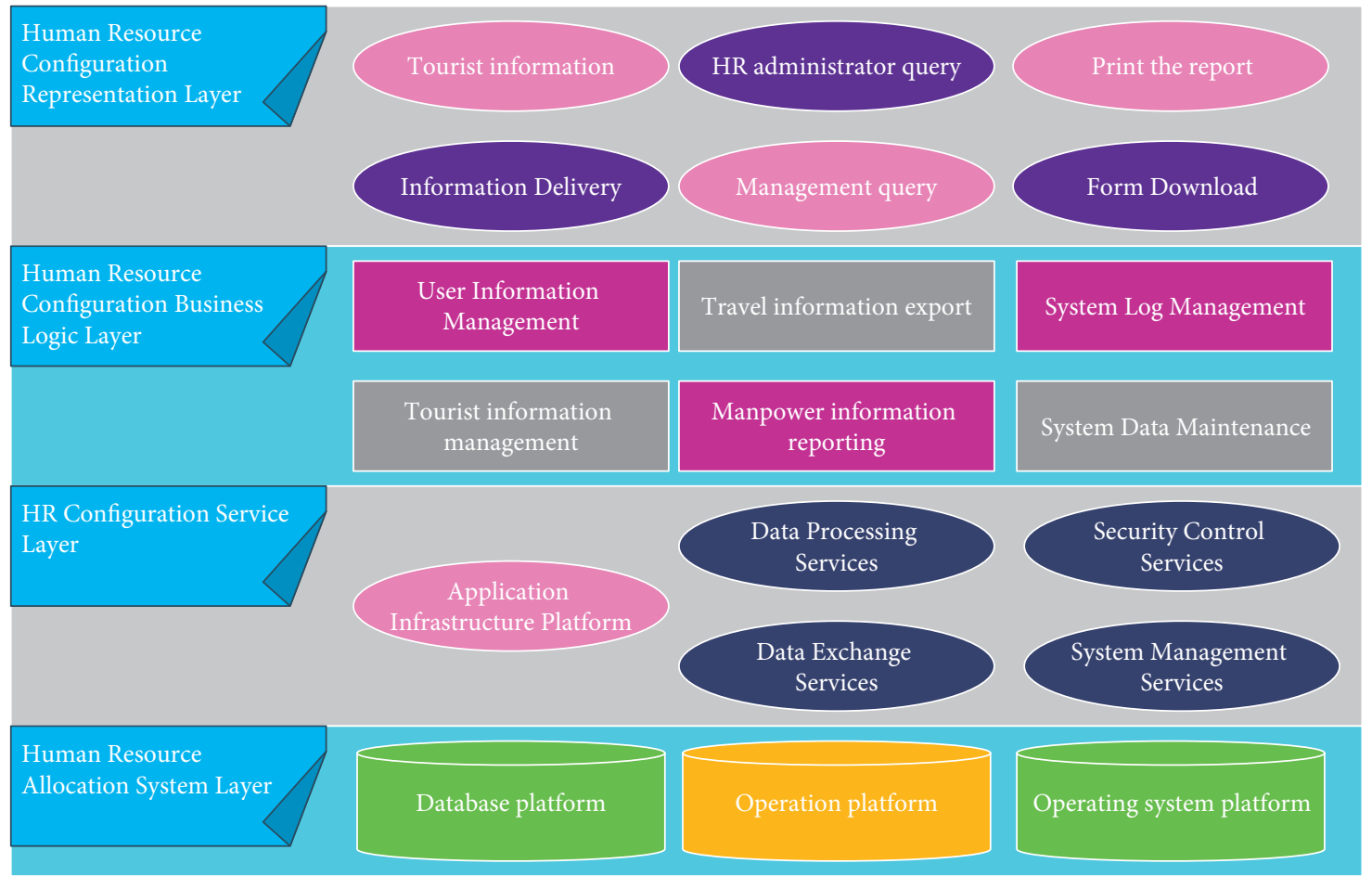

FIGURE 2: System architecture design diagram.

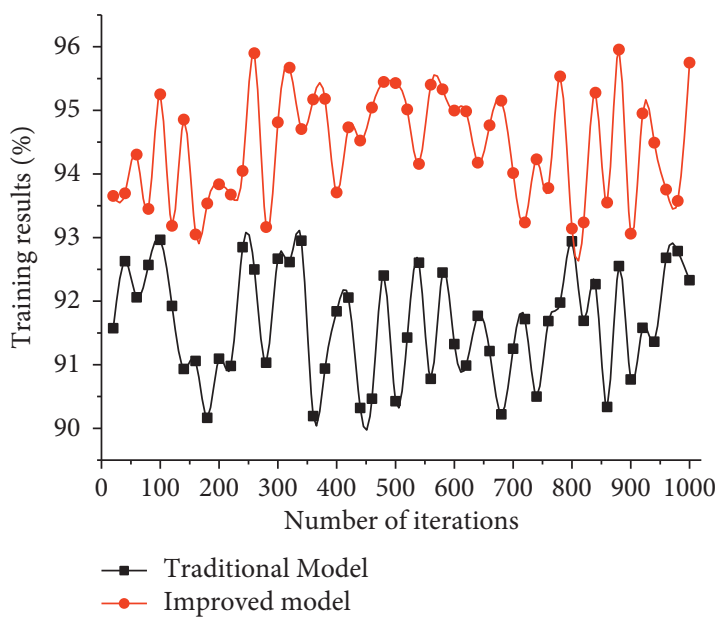

FIGURE 3: Comparison of models on EMNIST dataset.

convergence speed of the BP based on parameter random perturbation is significantly improved compared with that of the $\mathrm{BP}$ before improvement, and the convergence effect is shown in Figure 5. Compared with the traditional BP neural network, the convergence speed of the improved BP neural network algorithm is faster, and the convergence can achieve a better local optimal solution than the traditional BP. Although there are still fluctuations after the convergence smoothes out, the improved loss is still smaller than the loss before the improvement.

Compared with the BP before the improvement, the improved BP converges to be smoother after the loss is smaller and converges better, as shown in Figure 6. In this paper, the improved BP algorithm based on parameter

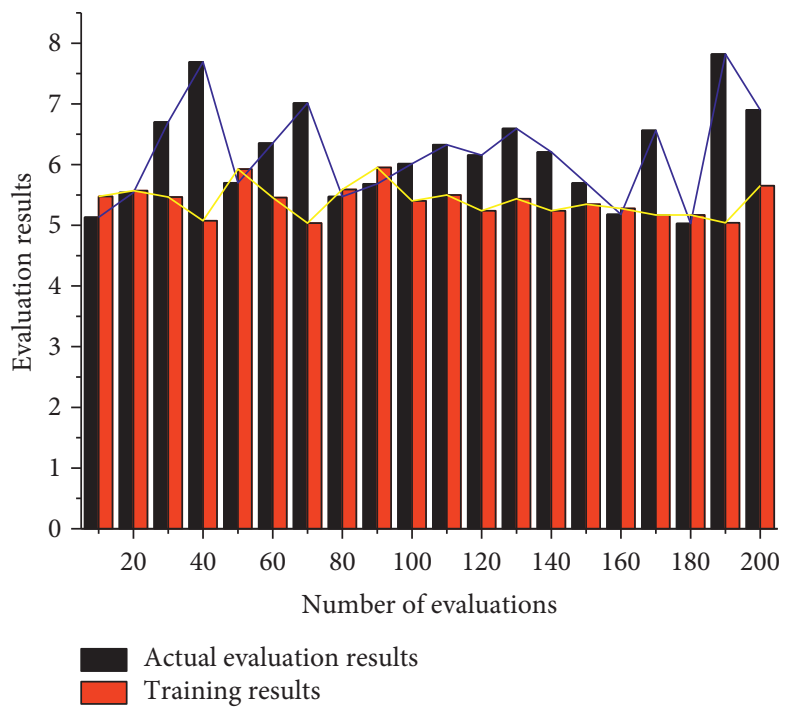

FIgURE 4: Model evaluation results.

random perturbation is proposed and the program design is given. The improved BP algorithm based on parameter random perturbation can converge faster and alleviate the gradient disappearance problem and the overfitting problem caused by the initial weight selection. Based on this, the actual prediction is carried out with stock prediction data as an example. The results show that the improved BP neural network can improve the accuracy of prediction compared with the traditional BP neural network. The random disturbance term is added to the connection weight of the input layer of the BP neural network 


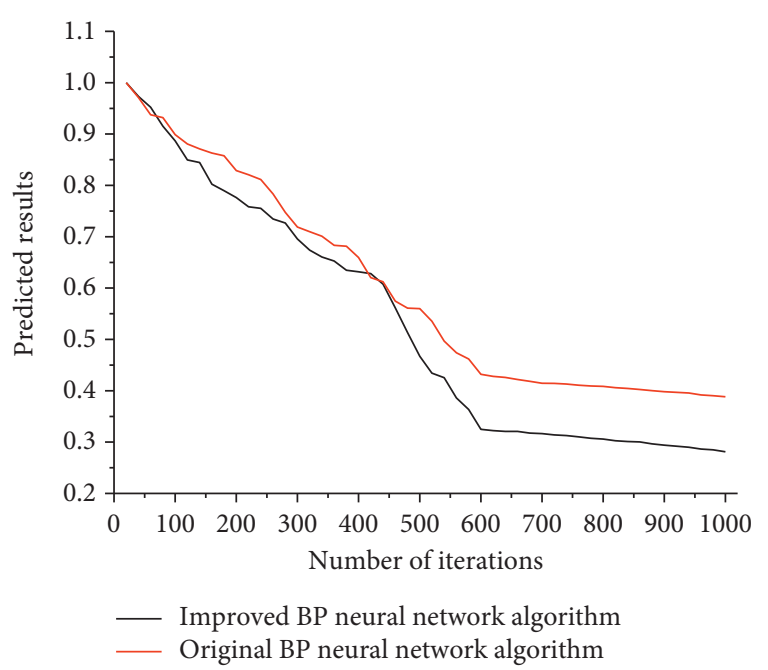

Figure 5: Comparison of the improved BP and the original BP prediction effect.

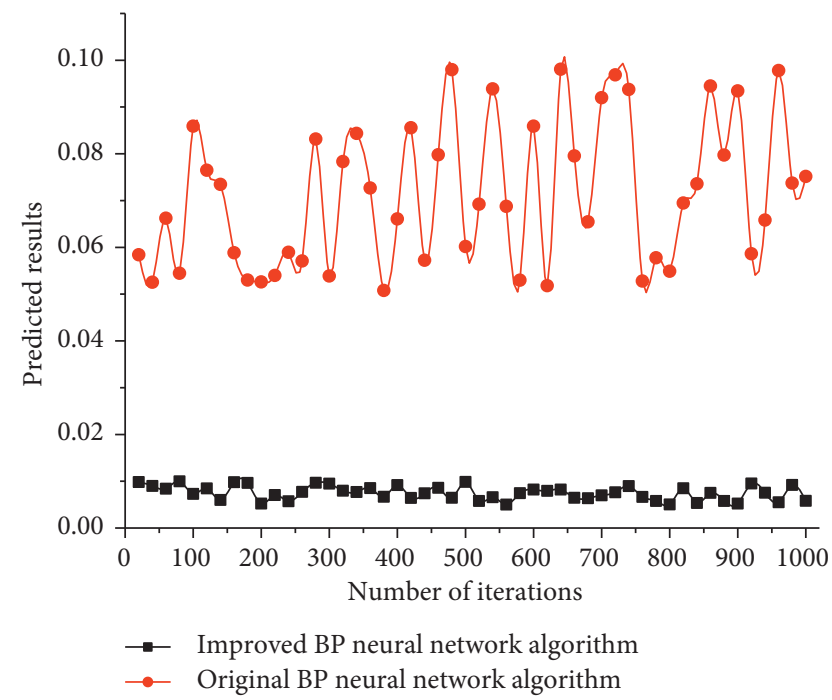

FIGURE 6: Comparison of prediction results after convergence and smoothing.

so that the improved BP neural network can converge faster. Use the extended library to construct an improved $\mathrm{BP}$ algorithm to manage the human resource configuration of a tourism industry. The experimental results show that compared with the original BP algorithm, the improved BP algorithm not only converges faster but also improves after the convergence is stable. The BP loss is smaller and the convergence effect is better.

\subsection{Human Resource Allocation System Evaluation Analysis.} The optimal total cost value is given in Figure 7, and the average cost is $\$ 132,312$. The optimal total cost value is $\$ 133,570$ when the dispersion of the robust parameters is 1 . It can be seen that further dispersion of the robust parameters enables the decision maker to better allocate manpower and reduce the total cost of the human resource allocation system. In addition, the standard deviation of these 20 group cost

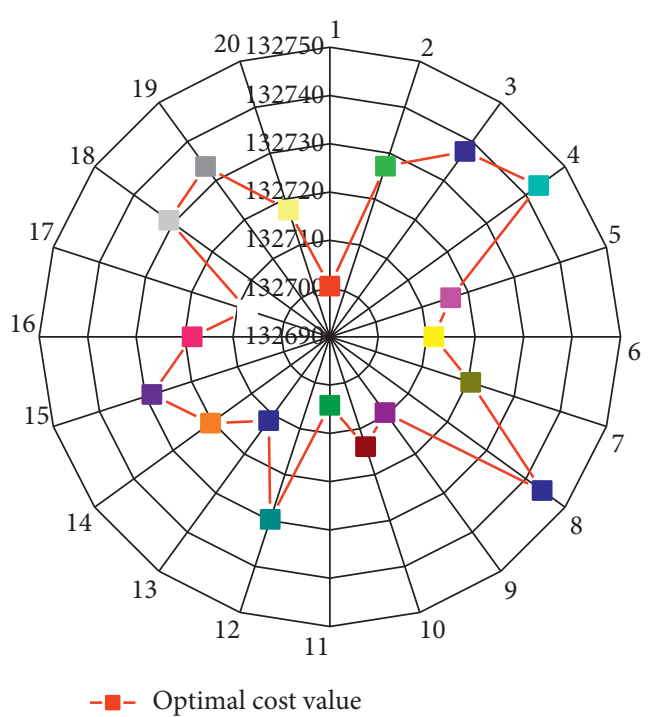

Figure 7: Optimal cost values for independent experiments.

values is 20.3 , which indicates that the designed human resource allocation system is stable. The comparison of the effects of the robust model and the deterministic model illustrates that the robust optimization method can resist the effects of customer arrival uncertainty on the system; also, in the most conservative time of the model, the penalty cost is 0 , i.e., no one is waiting in the queue at this time, the system has enough service personnel, and the total cost is much larger than the total cost in the optimal manpower case; thus, it can be seen that, in the deterministic model and the most conservative model, the total cost is greater than the value under the optimal robust parameters, indicating the effectiveness of the robust optimization method.

After the training satisfies the preset target requirements, the relevant training comparison values are obtained. As can be seen from Figure 8, the expected output values are very close to the values of the neural network training output values. In other words, the constructed improved BP neural network model can accurately determine the tourism competitiveness profile of smart tourism cities based on each evaluation index. Therefore, the network model training ends here, and the BP neural network-based tourism competitiveness evaluation model of wisdom tourism cities has been constructed. When evaluating the tourism competitiveness of other smart tourism cities, only the normalized data of the evaluation sample indicators need to be input to get the required evaluation conclusion. The maximum expected value is 0.5895 and the minimum is 0.3203 , which is in line with the experimental design. At the same time, the absolute error is in the range of 0.05 to 0.63 , and the relative error is only 0.1 to 0.37 . This value proves that the algorithm in this paper is more accurate.

Offline management not only refers to the daily management activities and operations of tourist attractions but also focuses on the correction and improvement of the real problems combined with online monitoring, online and offline as two channels, and interconnecting and interacting with each other; therefore, improving the management 

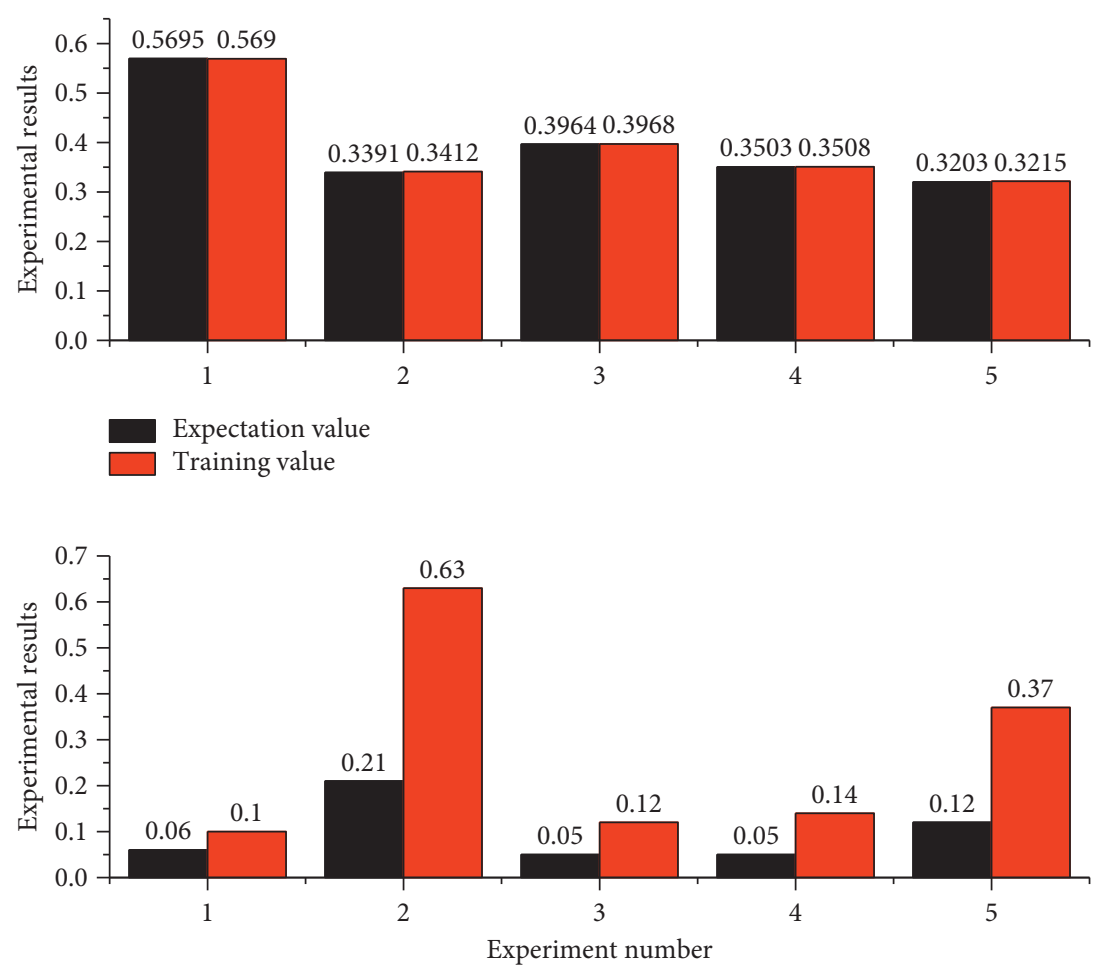

Absolute error Relative error

FIGURE 8: Expected output values versus training output values.

effectiveness of the actual work of tourist attractions, providing quality reception services and ecological scenic environment is not only the improvement of the management of tourist attractions but also an important way and means to promote the network of tourists, the important way and means of word-of-mouth publicity. Therefore, strengthening the offline management and enhancement of tourist attractions is an important foundation and prerequisite for shaping a good online image of tourist attractions as well as realizing the strategic plan for sustainable development of tourist attractions.

\section{Conclusion}

This paper shows that the combination of $\mathrm{BP}$ neural networks and various mathematical research methods has been extended to a wide range of research fields such as economy and society with high application value from the current state of research. Therefore, this paper makes use of the construction of the BP neural network and tries to apply it to the study of human resource allocation in the tourism industry based on the related literature research and theoretical analysis. From the perspectives of the nature of online texts, online text sources, and online text contents, the influencing factor model of tourism human resource allocation is constructed, and on this basis, the index system of tourism human resource allocation is proposed, mainly including three primary indicators of tourism resources, external environment, and management level and nine secondary indicators of natural resources, human resources, infrastructure, hospitality services, comprehensive management, political environment, and economic environment. The content of the specific web text is used as the index analysis element, and the basic attribute vocabulary commonly used in the web text about tourism scenic spots is compiled from two aspects: cognitive image and perceived image, which provides the basis for the collection of sample data. The model of tourism human resource allocation management is constructed as a dynamic structural combination consisting of an early warning system for information data collection and processing as the source and a response system for making crisis management decisions with cyclic feedback, which realizes the interpretation of the internal structural relationship of tourism human resource allocation management and outlines management priorities in three phases: latency, outbreak, and recovery periods. It proposes a systematic, dynamic, and institutionalized management approach for tourism human resource allocation; from the perspective of management operation, it proposes a procedure for tourism human resource allocation management and improves tourism human resource allocation program planning. Although the neural network algorithm applied in this thesis has certain advantages, selfadaptive ability, and learning ability and can be implemented in a relatively simple algorithm calculation using toolbox or language, but at the same time, there are certain limitations; the basis of the method requires the existence of a supervisory mechanism and positive and negative objective values in the process of data acquisition, which is a problem in this study. 


\section{Data Availability}

The data used to support the findings of this study are available from the corresponding author upon request.

\section{Conflicts of Interest}

The authors declare that they have no conflicts of interest or personal relationships that could have appeared to influence the work reported in this paper.

\section{Acknowledgments}

This work was supported by 2019 Research Project of Military-civilian Integration Development in Hebei Province: Research on the path of the development of military-civilian integration industry in Hebei Province under the background of "all elements, multiple fields, and high benefits" (HB19JMRH008).

\section{References}

[1] H. Xueqin, J. Ruimin, and W. Yaqi, "Research on Chengdu air cargo forecast based on improved ARIMA-GARCH," International Journal of Modelling in Operations Management, vol. 8, no. 3, pp. 299-312, 2021.

[2] Y. Wang and J. Chen, "Establishment of an economic value evaluation model of consumers' intention with marine tourism resources based on a backpropagation neural network," Journal of Coastal Research, vol. 108, no. SI, pp. 238-241, 2020.

[3] H. Wang and K. Islam, "An optimization model for poverty Alleviation fund audit mode based on BP neural network," Journal of Intelligent \& Fuzzy Systems, vol. 37, no. 1, pp. 481-491, 2019.

[4] W. Höpken, T. Eberle, and M. Fuchs, "Improving tourist arrival prediction: a big data and artificial neural network approach," Journal of Travel Research, vol. 60, no. 5, pp. 998-1017, 2021.

[5] A. Jahani and M. Saffariha, "Human activities impact prediction in vegetation diversity of lar national park in Iran using artificial neural network model," Integrated Environmental Assessment and Management, vol. 17, no. 1, pp. 42-52, 2021.

[6] C. Zhang, P. Patras, and H. Haddadi, "Deep learning in mobile and wireless networking: a survey," IEEE Communications Surveys \& Tutorials, vol. 21, no. 3, pp. 2224-2287, 2019.

[7] L. Wenwen, "Modeling and simulation of teaching quality in colleges based on BP neural network and training function," Journal of Intelligent \& Fuzzy Systems, vol. 37, no. 5, pp. 6349-6361, 2019.

[8] M. A. Hammad, B. Jereb, B. Rosi, and D. Dragan, "Methods and models for electric load forecasting: a comprehensive review," Logistics \& Sustainable Transport, vol. 11, no. 1, pp. 51-76, 2020.

[9] S. Shetaban, M. M. Seyyed Esfahani, and A. Saghaei, "Operations research and health systems: a literature review," Journal of Industrial Engineering and Management Studies, vol. 7, no. 2, pp. 240-260, 2020.

[10] S. Ronoud and S. Asadi, "An evolutionary deep belief network extreme learning-based for breast cancer diagnosis," Soft Computing, vol. 23, no. 24, pp. 13139-13159, 2019.
[11] A. Aakash, A. Tandon, and A. Gupta Aggarwal, "How features embedded in eWOM predict hotel guest satisfaction: an application of artificial neural networks," Journal of Hospitality Marketing \& Management, vol. 30, no. 4, pp. 486-507, 2021.

[12] L. A. Ocampo, G. K. M. Abad, and K. G. L. Cabusas, "Recent approaches to supplier selection: a review of literature within 2006-2016," International Journal of Integrated Supply Management, vol. 12, no. 1-2, pp. 22-68, 2018.

[13] B. Huang and H. Hao, "A novel two-step procedure for tourism demand forecasting," Current Issues in Tourism, vol. 24, no. 9, pp. 1199-1210, 2021.

[14] X. Cheng and C. Zhao, "Prediction of tourist flow based on deep belief network and echo state network," Revue d'Intelligence Artificielle, vol. 33, no. 4, pp. 275-281, 2019.

[15] L. Kang, W. Zhu, and G.-G. Yoon, "VR design of public facilities in historical blocks based on BP neural network," Neural Processing Letters, vol. 53, no. 4, pp. 2457-2466, 2021.

[16] W. Dai and P. Hu, "Application of BP neural network in the analytic hierarchy process of person-post evaluation model," The Journal of Supercomputing, vol. 76, no. 2, pp. 897-914, 2020.

[17] Y. Chen, "A survey on industrial information integration 2016-2019," Journal of Industrial Integration and Management, vol. 5, no. 1, pp. 33-163, 2020.

[18] C. Shi and Z. Zhang, "A prediction method of regional water resources carrying capacity based on artificial neural network," Earth Sciences Research Journal, vol. 25, no. 2, pp. 169-177, 2021.

[19] Y. Zhou, F. R. Yu, and J. Chen, "Cyber-physical-social systems: a state-of-the-art survey, challenges and opportunities," IEEE Communications Surveys \& Tutorials, vol. 22, no. 1, pp. 389-425, 2019.

[20] Z. Tong, F. Ye, M. Yan, H. Liu, and S. Basodi, "A survey on algorithms for intelligent computing and smart city applications," Big Data Mining and Analytics, vol. 4, no. 3, pp. 155-172, 2021. 